\title{
Multifocal aggressive angiomyxoma: a case report
}

\author{
A Kaur, P S Makhija, E Vallikad, V Padmashree, H S Indira
}

\begin{abstract}
A case of aggressive angiomyxoma in a 25 year old woman is presented. The patient was admitted to hospital with a history of hesitancy of micturation and pain in the right iliac fossa. She was found to have a left labial mass, which was clinically diagnosed to be a Bartholin gland cyst. A pelvic ultrasound revealed an additional mass in the right paravesical region. At surgery, two distinct masses were removed, one from the right perivesical space and the other from the left labium. Both masses were rubbery, white, and gelatinous and showed similar histopathology findings of thick and thin walled vascular channels set in a loose myxoid stroma. A diagnosis of multifocal aggressive angiomyxoma was made. This is the first reported case of aggressive angiomyxoma occurring as two distinct masses in one patient (f Clin Pathol 2000;53:798-799)
\end{abstract}

Keywords: aggressive angiomyxoma; myxoma; myxoid tumours; aggressive angiomyxoma of female pelvis; female perineum

Aggressive angiomyxoma was first reported as a distinct variant of myxoid neoplasms in the female vagina and pelvis by Steeper and Rosai. ${ }^{1}$ These lesions have a predilection for female pelvic soft parts, slow growth, frequent recurrences, and are characterised histologically by a predominantly myxoid stroma and an abundance of thin and thick walled vascular channels. Many authors have subsequently reported this lesion in female and male patients. In our paper, we report the first case of aggressive angiomyxoma occurring in two different locations in the same patient.

Department of Pathology, St Johns Medical College and Hospital, Sarjapur Road Bangalore, 560034 Karnataka, India

A Kaur

P S Makhija

Department of Gynaecology, St Johns

Medical College and Hospital

E Vallikad

V Padmashree

H S Indira

Correspondence to: Dr Makhija pmakhija@satyam.net.in

Accepted for publication 20 January 2000

A 25 year old woman presented with mild pain in the right iliac fossa and hesitancy of micturation on and off for three months. On examination, she was a moderately built and nourished woman. Systemic examination was normal. Vaginal examination showed a left labial mass measuring $4 \times 4 \mathrm{~cm}$, and clinically thought to be a Bartholin gland cyst. The cervix and vagina were healthy. The uterus was normal in size, pushed to the left and posteriorly with restricted mobility. There was fullness in the right fornix. Another soft mass was palpable on the right side of the uterus, which was mobile and non-tender.

Clinically, a tentative diagnosis of a left sided ovarian mass with a right Bartholin cyst was made. On laparoscopy, both ovaries were visualised and were grossly normal. A soft irregular

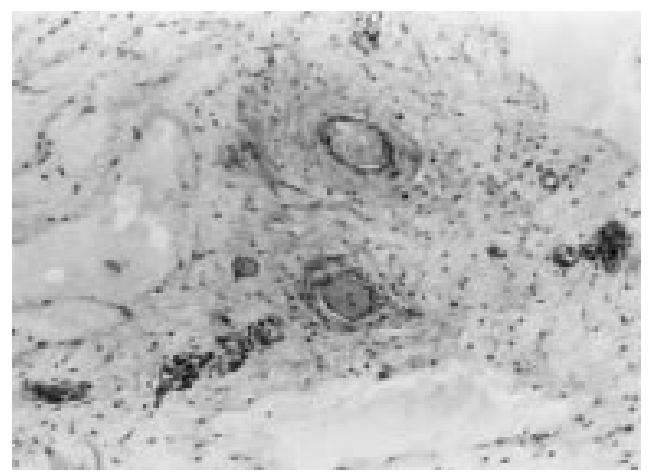

Figure 1 Section from the vulvar mass showing myxoid areas with stellate cells and blood vessels (haematoxylin and eosin stained; magnification, $\times 100$ ).

mass was seen arising from the soft tissue in the paravesical space, measuring $25 \times 20 \mathrm{~cm}$, seen lateral to the bladder on the right side. Retroperitoneally, it was seen extending deep into the paravesical space, posterior to the inguinal ligament near the base of the femoral triangle. It was very close to the posterior surface of the symphysis pubis and superior ramus of the pubic bone. The tumour appeared to be encapsulated and was separable from the surrounding tissue, with few feeding blood vessels. The entire mass was removed and sent for histopathological examination along with the smaller vulvar mass. The larger pelvic mass was found to measure $14 \times 10 \times 8 \mathrm{~cm}$ and weigh $490 \mathrm{gm}$, whereas the smaller vulvar mass measured $12 \times 4 \times 3 \mathrm{~cm}$.

On sectioning, both the masses had focally infiltrating margins and a rubbery glistening grey/white surface. Several representative samples were taken. Microscopically, both the tumours had similar features. Numerous thin and thick walled blood vessels of varying calibre were noted along with spindle shaped and stellate neoplastic cells in a myxoid to collagenous stroma (figs 1 and 2). Extravasation of red blood cells was seen in some areas. A diag-
Figure 2 Section from the pelvic mass showing stellate cells in a myxoid stroma with prominent blood vessels.

(haematoxylin and eosin stained; magnification, $\times 100$ ).

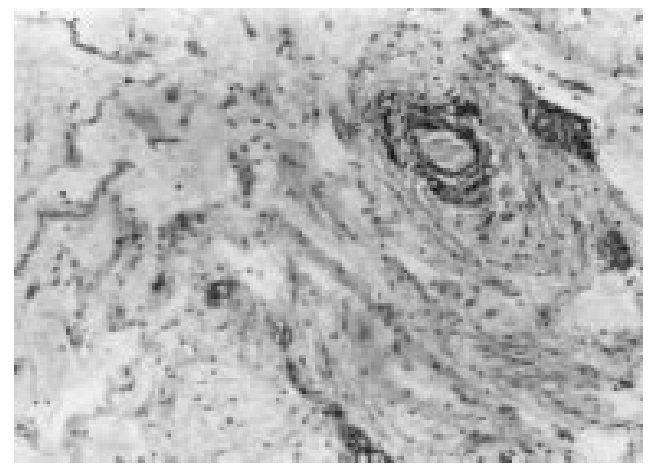


nosis of multifocal aggressive angiomyxoma was made. The patient has had no recurrences eight months after surgery and has been coming regularly for follow up.

\section{Discussion}

Aggressive angiomyxoma is a rare but well described neoplasm. Steeper and Rosai first recognised it as a separate histopathological entity in 1983. This is a distinctive tumour with a characteristic clinical course and specific gross and microscopic features. Although most reported cases are in women (with a predilection for pelvic soft parts, vagina, vulva, and more rarely buttocks, retroperitoneum, and the inguinal region), a small number have been reported in men. ${ }^{2}$ Aggressive angiomyxomas in men occur over a wide variety of sites around the genital tract such as perineum, perianal region, scrotum, spermatic cord, inguinal region, and pelvic soft tissues. ${ }^{3}$

Clinically, this tumour is most frequently diagnosed as a vulvar mass, vulvar abscess, Bartholin cyst, Gartners duct cyst, vaginal cyst, and a levator or perineal hernia. ${ }^{4}$ The final diagnosis is usually provided by the pathologist. Aggressive angiomyxoma occurs in ages ranging from 15 to 63 years. ${ }^{4}$ In both male and female patients, aggressive angiomyxoma presents as a polypoid or a cyst like lesion, or as an ill defined swelling in the pelvic region with infiltrating margins. It is characteristically a slow growing, focally infiltrative tumour, lacking a capsule.

On gross examination these tumours are soft, bulky masses with a homogenous glistening cut surface. Histologically, the tumour shows vascular channels of varying sizes distributed haphazardly in the myxoid stroma. Nuclear atypia and mitosis are not present.

The differential diagnoses ranges from benign tumours such as myxolipoma, myxoid neurofibroma, and myxoid leiomyoma to myxofibrosarcoma, myxoid liposarcoma, and botryoid rhabdomyosarcoma. ${ }^{367}$ This neoplasm may also be misdiagnosed as vaginal polyps, myxoma, spindle cell lipoma, and fibromatosis. The distinctively striking vascular component in aggressive angiomyxoma helps in ruling out most of the above mentioned neoplasms as differentials. Aggressive angiomyxoma in the perineum may also be misdiagnosed as angiomyofibroblastoma. ${ }^{8}$ However, the latter has circumscribed margins, higher cellularity, plumper stromal cells, and many more vascular channels. Another important entity, which has to be kept in mind especially in the case of multifocal tumours, as in our case, is disseminated peritoneal leiomyomatosis, ${ }^{9}$ which can be associated with multiple leiomyomas in the uterus and pelvis. Our present case had no uterine leiomyomas on the pelvic scan. The prominent vascularity, along with the paucity of smooth muscle fibres in the present lesion, helped to rule out multi- focal leiomyomas. Of the malignant neoplasms, myxoid liposarcomas may rarely be multifocal. ${ }^{10}$ The absence of lipoblasts in aggressive angiomyxoma helps to differentiate it from a liposarcoma.

The bland histology and the lack of reported metastases after long periods of follow up favour a benign nature of this neoplasm. However, this tumour is locally invasive and tends to infiltrate deep into pelvic soft tissues. It is also associated with frequent recurrences, probably secondary to incomplete removal. The criteria that reliably predict the behavior of these lesions are not known, and recurrences have been observed in small lesions and in neoplasms with negative surgical margins.

Aggressive angiomyxomas occurring in the vulva have been reported to recur after excision, in the ischiorectal space and retroperitoneum. However, the occurrence of this tumour as two discrete masses in the same patient has not been reported to date. In our case, two distinct masses were present, one in the left labium and the other in the right paravesical space. The two masses had a similar gross and microscopic appearance. This brings to light the fact that these neoplasms can have a multifocal occurrence, which might explain the recurrences in some of the neoplasms, especially those with negative surgical margins.

In conclusion, aggressive angiomyxoma is a rare, benign neoplasm that can be mistaken both clinically and on microscopy for several other conditions. It is important to diagnose this condition because the tumour is locally infiltrative and requires wide excision and follow up. Our report highlights the possibility of a multifocal presentation of aggressive angiomyxoma, thus emphasising the need for a complete investigation in all cases of aggressive angiomyxoma, and a careful search for additional masses to exclude multifocality.

1 Steeper TA, Rosai J. Aggressive angiomyxoma of the pelvis and perineum: report of nine cases of a distinctive type of gynaecologic soft tissue neoplasm. Am $\mathcal{F}$ Clin Pathol 1983;7:453.

2 Begin LR, Clement PB, Kirk ME, et al. Aggressive angiomyxoma of pelvic soft parts: a clinicopathologic study of nine cases. Hum Pathol 1985;16:621-8.

3 Tsang WY. Aggressive angiomyxoma a report of four cases occurring in men. Am $\mathcal{F}$ Surg Pathol 1992;16:1059-65.

4 Simo M, Zapata C, Esquius J, et al. Aggressive angiomyxoma of the female pelvis and perineum. Report of two
my myxoma of the female pelvis and perineum. Report of two cases and review

5 Woods SD, Essex WB, Hughes ESR, et al. Aggressive angiomyxoma of the female pelvis. Aust NZ F Surg 1987;57:6878.

6 Enzinger FM, Weiss SW. Soft tissue tumors, 3rd ed. St Louis: CV Mosby, 1995.

7 Stephenson BM, Williams EV. Aggressive angiomyxoma of the female perineum and pelvis. BrF Surg 1992;79:1181.

8 Fletcher CDM, Tsang WY, Lee KC, et al. Angiomyofibroblastoma of the vulva. A benign neoplasm distinct from goressive angiomyxoma. Am f Surg Pathol 1992;16:37382 .

9 Valente PT. Leiomyomatosis peritonealis disseminata. A report of two cases and review of the literature. Arch Pathol Lab Med 1984;108:669-72.

10 Seenu V, Kriplani AK, Shukla NK, et al. Multicentric liposarcoma: report of two cases. Surg Today 1995;25:447- 